University of Texas at El Paso

ScholarWorks@UTEP

$7-2014$

\title{
From Mean and Median Income to the Most Adequate Way of Taking Inequality Into Account
}

Vladik Kreinovich

The University of Texas at El Paso, vladik@utep.edu

Hung T. Nguyen

New Mexico State University - Main Campus, hunguyen@nmsu.edu

Rujira Ouncharoen

Chiang Mai University, rujira.o@cmu.ac.th

Follow this and additional works at: https://scholarworks.utep.edu/cs_techrep

Part of the Econometrics Commons

Comments:

Technical Report: UTEP-CS-14-39a

Published in: Van-Nam Huynh, Vladik Kreinovich, Songsak Sriboonchitta, and Komsan Suriya (eds.), Econometrics of Risk, Springer Verlag, Berlin, Heidelberg, 2015, pp. 63-73.

\section{Recommended Citation}

Kreinovich, Vladik; Nguyen, Hung T.; and Ouncharoen, Rujira, "From Mean and Median Income to the Most Adequate Way of Taking Inequality Into Account" (2014). Departmental Technical Reports (CS). 850.

https://scholarworks.utep.edu/cs_techrep/850

This Article is brought to you for free and open access by the Computer Science at ScholarWorks@UTEP. It has been accepted for inclusion in Departmental Technical Reports (CS) by an authorized administrator of ScholarWorks@UTEP. For more information, please contact Iweber@utep.edu. 


\title{
From Mean and Median Income to the Most Adequate Way of Taking Inequality Into Account
}

Vladik Kreinovich, Hung T. Nguyen, and Rujira Ouncharoen

\begin{abstract}
How can we compare the incomes of two different countries or regions? At first glance, it is sufficient to compare the mean incomes, but this is known to be not a very adequate comparison: according to this criterion, a very poor country with a few super-rich people may appear to be in good economic shape. A more adequate description of economy is the median income. However, the median is also not always fully adequate: e.g., raising the income of very poor people clearly improves the overall economy but does not change the median. In this paper, we use known techniques from group decision making - namely, Nash's bargaining solution - to come up with the most adequate measure of "average" income: geometric mean. On several examples, we illustrate how this measure works.
\end{abstract}

\section{Mean Income, Median Income, What Next?}

Mean income and its limitations. At first glance, if we want to compare the economies of two countries or two regions, all we need to do is divide, for each country, the total income by the number of people and compare the resulting values of mean income. If the mean income in country $A$ is larger than the mean income

Vladik Kreinovich

Department of Computer Science, University of Texas at El Paso, 500 W. University, El Paso, TX 79968, USA, e-mail: vladik@utep.edu

Hung T. Nguyen

Department of Mathematical Sciences, New Mexico State University, Las Cruces, New Mexico 88003, USA, and Faculty of Economics, Chiang Mai University, Chiang Mai, Thailand, e-mail: hunguyen@nmsu.edu

Rujira Ouncharoen

Department of Mathematics, Chiang Mai University, Chiang Mai, Thailand, e-mail: rujira.o@cmu.ac.th 
in country $B$, this means that the economy of country $\mathrm{A}$ is in better shape than the economy of country B.

In many cases, this conclusion is indeed justified, but not always. The fact that the mean has limitations can be illustrated by a known joke: "What happens when Bill Gates walks into a bar? On average, everyone becomes a millionaire.” This is a joke, but this joke reflects a serious problem: if a billionaire moves into a small and very poor country, the mean income in this country would increase but the country would remain very poor, contrary to the increase in the mean.

In other words, when comparing different economies, we need to take into account not only the total income, but also the degree of inequality in income distribution.

Comment. In technical terms, we would like the proper measure of "average" income to not change much if we add of an outlier like Bill Gates. In statistics, the corresponding property of statistical estimates is known as robustness; see, e.g., $[9,29]$. In these terms, the main problem of the mean is that it is not robust.

Medium income: a more adequate measure. To avoid the above problem, economists proposed several alternatives to the mean income. The most widely used alternative is the median income, i.e., the income level for which the income of exactly half of the population is above this level - and the income of the remaining half is below this level. For example, this is how the Organization for Economic Cooperation and Development (OECD) compares economies of different countries: by listing both their mean incomes and their median incomes; see, e.g., [23].

Median resolves some of the problems related to mean: for example, when Bill Gates walks into a bar, the mean income of people in the bar changes drastically, but the median does not change much.

Comment. The main problem with the mean, as we have mentioned, is that the mean is not robust. From this viewpoint, median - a known robust alternatives to the mean $[9,29]$ - seems a reasonable replacement of the mean.

Limitations of the median and remaining practical problem. While the median seems to be a more adequate measure of "average" income than the mean, it is not a perfect measure. For example, if the incomes of all the people in the poorer half increase - but do not exceed the previous median - the median remains the same. This is not a very adequate measure for governments that try to lift people out of poverty. Similarly, if the income of the poorer half drastically decreases, we should expect the adequate measure of "average" income to decrease - but the median remains unchanged.

Comment. After we reformulated the problem with mean in terms of robustness, a reader may be under the impression that robustness is all we seek. Alas, the above limitation shows that the problem of finding an appropriate measure of "average" income goes beyond robustness; namely:

- the main problem of mean is that it is not robust - it changes too much when we would like to change it a little bit; 
- however, while the median is robust, it has another problem - it is "too robust": it changes too little (actually, not at all) when we would like it to change.

This example shows that we cannot solve our problem by simply reducing it to a known statistical problem of designing robust estimates, we do not need to solve the original problem of estimating the "average" income.

How this practical problem is resolved now. At present, economists propose different heuristic measures of "average" income which are supposedly more adequate than mean and median. There is no absolutely convincing arguments in favor of this or that measure; as a result, researchers use emotional and ideological arguments; see, e.g., [24].

What we do in this paper. In this paper, we show that under some reasonable conditions, it is possible to find the most adequate way how to take inequality into account when gauging the "average" income.

\section{Analysis of the Problem and the Resulting Measure}

The problem of gauging "average" income can be viewed as a particular case of a problem of group decision making. For the problem of gauging "average" income - when taken "as is" - there is no immediate solution yet. Let us show, however, that this gauging problem can be reformulated in terms of a problem for which many good solutions have been developed - namely, the problem of group decision making.

To explain this reformulation, let us start with the simplest possible case our main problem: the case when in each of the two compared regions, there is perfect equality: all the people in the first region have the same income $x$, and all the people in the second region have the same income $y$. In this case clearly:

- if $x>y$, this means that the first region is in better economic shape, and

- if $x<y$, this means that the second region is in better economic shape.

What if we consider a more realistic case of inequality, when people in the first region have, in general, different incomes $x_{1}, \ldots, x_{n}$, and people in the second area also have, in general, different incomes $y_{1}, \ldots, y_{m}$ ? How can we then compare the two regions?

A natural idea is to reduce this comparison to the case when all the incomes are equal. In other words:

- first, we find the value $x$ such that for the group of all the people from the first region, incomes $x_{1}, \ldots, x_{n}$ are equivalent - in terms of group decision making to all of them getting the same income $x$;

- then, we find the value $y$ such that for the group of all the people from the second region, incomes $y_{1}, \ldots, y_{n}$ are equivalent - in terms of group decision making to all of them getting the same income $y$; 
- finally, we compare the resulting values $x$ and $y$ : if $x>y$, then the first economy is in better shape, otherwise, if $x<y$, the second economy is in better shape.

Comment. Our main idea is to reduce the econometric problem of finding an adequate measure for "average" income" to a game-theoretic problem of cooperative group decision making. This idea is in line with the emerging view that game theory - which was originally invented as a general theory of group behavior - should be (and can be) successfully applied not only to situations of conflicting competition, but also to more general problems of economics - in particular, to problems of financial econometrics.

From the idea to the algorithm. To transform the above idea to the algorithm, let us recall a reasonable way to perform group decision making. In group decision making, we need to order situations with different individual incomes. To be more precise, in group decision making, we consider situations with different individual utility values $u_{1}, \ldots, u_{n}$ - since different people value different income levels differently; see, e.g., [6, 14, 22, 26]. In this case, as shown by the Nobelist John Nash, under some reasonable assumptions, the most adequate solution is to select the alternative for which the product of the utilities $\prod_{i=1}^{n} u_{i}$ is the largest possible; see, e.g., $[14,21,26]$.

The utility is usually proportional to a power of the money: $u_{i}=C_{i} \cdot x_{i}^{a}$ for some $a \approx 0.5$; see, e.g., $[11,12,13]$. Substituting these utility values into Nash's formula, we get the product $\prod_{i=1}^{n} C_{i} \cdot \prod_{i=1}^{n} x_{i}^{a}$. In these terms, to find the value $x$ for which the selection $\left(x_{1}, \ldots, x_{n}\right)$ is equivalent to $x$, we must find $x$ for which

$$
\prod_{i=1}^{n} C_{i} \cdot \prod_{i=1}^{n} x_{i}^{a}=\prod_{i=1}^{n} C_{i} \cdot \prod_{i=1}^{n} x^{a} .
$$

Dividing both sides of this equality by the constant $\prod_{i=1}^{n} C_{i}$ and extracting power $a$ from both sides, we conclude that $\prod_{i=1}^{n} x_{i}=\prod_{i=1}^{n} x=x^{n}$. Thus, the value $x$ which describes the income distribution $\left(x_{1}, \ldots, x_{n}\right)$ is equal to $x=\sqrt[n]{x_{1} \cdot \ldots \cdot x_{n}}$ - the geometric mean of the income values. So, we arrive at the following conclusion.

Resulting measure of "average" income which most adequately described "average" income: geometric mean. Suppose that we need to compare the economies of two regions. Let us denote the incomes in the first region by $x_{1}, \ldots, x_{n}$ and the incomes in the second region by $y_{1}, \ldots, y_{m}$. To perform this comparison, we compute the geometric averages $x=\sqrt[n]{x_{1} \cdot \ldots \cdot x_{n}}$ and $y=\sqrt[m]{y_{1} \cdot \ldots \cdot y_{m}}$ of the two regions; then:

- if $x>y$, we conclude that the first region is in better economic shape, and

- if $x<y$, we conclude that the second region is in better economic shape. 
From the mathematical viewpoint, comparing geometric means $\bar{x}$ and $\bar{y}$ is equivalent to comparing the logarithms of these means. Here,

$$
\ln (\bar{x})=\ln \left(\sqrt[n]{x_{1} \cdot \ldots \cdot x_{n}}\right)=\frac{\ln \left(x_{1}\right)+\ldots+\ln \left(x_{n}\right)}{n} .
$$

Thus, the logarithm of the geometric mean $\bar{x}$ is equal to mean value $E[\ln (x)]$ of the logarithm of the income - and therefore,

$$
\bar{x}=\exp (E[\ln (x)])=\exp \left(\int \ln (x) \cdot f(x) d x\right) .
$$

So, to compare the economies in two different regions, we need to compare the mean values $E[\ln (x)]$ of the logarithm of the income $x$ in these regions.

Relation between the new measure and the mean income: an observation. It is well known that the geometric mean is always smaller than or equal to the arithmetic mean, and they are equal if and only if all the numbers are equal; see, e.g., [1, 32].

Thus, the new measure of "average" income is always smaller than or equal that the mean income, and it is equal to the mean income if and only if all the individual incomes are the same - i.e., if and only if we have perfect equality.

\section{First Example of Using the New Measure of "Average" Income: Case of Low Inequality}

Case of low inequality: informal description. Let us first consider the case when inequality is low, i.e., when most people have a reasonable income, and the proportion of very poor and very rich people is not that large.

Towards a formal description. The fact that most incomes are close to one another means that most of these incomes are close to the mean income $\mu$. In mathematical statistics, deviations from the mean are usually described by the standard deviation $\sigma$; see, e.g., [30]. In these terms, low inequality means that the standard deviation $\sigma$ is small. Let us analyze what happens in this case.

Case of low inequality: analysis of the problem. As we have mentioned, the new inequality measure has the form $\bar{x}=\exp (E[\ln (x)])$. Thus, to compare the economies in two different regions, we need to compare the mean values $E[\ln (x)]$ of the logarithm of the income $x$ in these regions.

Since the deviations from the mean $x-\mu$ are relatively small, we have can substitute $x=\mu+(x-\mu)$ into the formula for $E[\ln (x)]$ and ignore higher order terms in the expansion in $x-\mu$. According to the Taylor series for the logarithm, we have:

$$
\ln (x)=\ln (\mu+(x-\mu))=\ln (\mu)+\frac{1}{\mu} \cdot(x-\mu)-\frac{1}{2 \mu^{2}} \cdot(x-\mu)^{2}+\ldots
$$


By taking the mean value of both sides and taking into account that $E[x-\mu]=$ $\mu-\mu=0$ and that $E\left[(x-\mu)^{2}\right]=\sigma^{2}$, we conclude that

$$
E[\ln (x)]=\ln (\mu)-\frac{1}{2 \mu^{2}} \cdot \sigma^{2}+\ldots
$$

Since we assumed that the deviations of $x$ from $\mu$ are small, we can preserve only the first terms which shows the dependence on these deviations and ignore higher order terms in this expansion. As a result, we get an approximate formula

$$
E[\ln (x)] \approx \ln (\mu)-\frac{\sigma^{2}}{2 \mu^{2}} .
$$

Thus, for $\bar{x}=\exp (E[\ln (x)])$, we get

$$
\bar{x}=\exp (E[\ln (x)]) \approx \exp \left(\ln (\mu)-\frac{\sigma^{2}}{2 \mu^{2}}\right)=\exp (\ln (\mu)) \cdot \exp \left(-\frac{\sigma^{2}}{2 \mu^{2}}\right) .
$$

The first factor is equal to $\mu$. To estimate the second factor, we can again use the fact that $\sigma$ is small; in this case, we can expand the function $\exp (z)$ in Taylor series and keep only the first term depending on $\sigma$ :

$$
\exp \left(-\frac{\sigma^{2}}{2 \mu^{2}}\right)=1-\frac{\sigma^{2}}{2 \mu^{2}}+\ldots \approx 1-\frac{\sigma^{2}}{2 \mu^{2}} .
$$

Substituting this expression into the above formula for $\bar{x}=\exp (E[\ln (x)])$, we conclude that

$$
\bar{x}=\mu \cdot\left(1-\frac{\sigma^{2}}{2 \mu^{2}}\right)=\mu-\frac{\sigma^{2}}{2 \mu} .
$$

Thus, we arrive at the following conclusion.

Resulting formula. In the case of low inequality, the "average" income is equal to

$$
\bar{x}=\mu-\frac{\sigma^{2}}{2 \mu},
$$

where $\mu$ is the average income and $\sigma$ is the standard deviation.

Analysis of this formula. The larger the inequality, the larger the standard deviation $\sigma$, and the less preferable is the economy. The above formula provides an exact quantitative description of this natural qualitative idea.

Comments.

- The new measure takes inequality into account, and it avoids the ideological ideas of weighing inequality too much: if an increase in the mean income comes at the expense of an increase in inequality, this is $\mathrm{OK}$, as long as the above combination of means and standard deviation increases. 
- This example is one of the cases which shows that the new measure is more adequate than, e.g., the median. For example, if the incomes are normally distributed, then the median simply coincides with the mean, and so, contrary to our intuitive expectations, the increase in inequality does not worse the median measure of economics. In contrast, the new measure does go down when inequality increases.

\section{Second Example of Using the New Measure of "Average" Income: Case of a Heavy-Tailed Distribution}

Heavy-tailed (usually, Pareto) distributions are ubiquitous in economics. In the 1960s, Benoit Mandelbrot, the author of fractal theory, empirically studied the price fluctuations and showed [15] that large-scale fluctuations follow the Pareto powerlaw distribution, with the probability density function $f(x)=A \cdot x^{-\alpha}$ for $x \geq x_{0}$, for some constants $\alpha \approx 2.7$ and $x_{0}$. For this distribution, variance is infinite. The above empirical result, together with similar empirical discovery of heavy-tailed laws in other application areas, has led to the formulation of fractal theory; see, e.g., $[16,17]$.

Since then, similar Pareto distributions have been empirically found in other financial situations $[3,4,5,7,18,20,25,28,31,33,34]$, and in many other application areas $[2,8,16,19,27]$.

Formulation of the problem. Let us consider the situations when the income distribution follows Pareto law, with probability density $f(x)$ equal to 0 for $x \leq x_{0}$ and to $A \cdot x^{-\alpha}$ for $x \geq x_{0}$.

Once we know $x_{0}$ and $\alpha$, we can determine the parameter $A$ from the condition that $\int f(x) d x=1$. For the above expression, this condition leads to $A \cdot \frac{x_{0}^{-(\alpha-1)}}{\alpha-1}=1$, hence $A=(\alpha-1) \cdot x_{0}^{\alpha-1}$.

For this distribution, we want to compute the mean income, the median income, and the newly defined "average" income.

Mean income. The mean income is equal to $\mu=\int x \cdot f(x) d x$, i.e., for the Pareto distribution, to

$$
\int_{x_{0}}^{\infty} A \cdot x^{1-\alpha} d x=\left.A \cdot \frac{x^{2-\alpha}}{2-\alpha}\right|_{x_{0}} ^{\infty}=A \cdot \frac{x_{0}^{2-\alpha}}{\alpha-2}
$$

Substituting the above value of $A$, we conclude that the mean is equal to

$$
\mu=\frac{\alpha-1}{\alpha-2} \cdot x_{0}
$$

Median income. The median income $m$ can be determined from the condition that $\int_{m}^{\infty} f(x) d x=\frac{1}{2}$. For the Pareto distribution, this means 


$$
\int_{m}^{\infty} A \cdot x^{-\alpha} d x=A \cdot \frac{m^{-(\alpha-1)}}{\alpha-1}=\frac{1}{2} \text {. }
$$

Substituting the above expression for $A$ into this formula, we conclude that $\frac{m^{-(\alpha-1)}}{x_{0}^{-(\alpha-1)}}=$ $\frac{1}{2}$, hence $\frac{m^{\alpha-1}}{x_{0}^{\alpha-1}}=2$, and $m=x_{0} \cdot 2^{1 /(\alpha-1)}$.

New measure of "average" income. For the new measure of average income $\bar{x}$, its logarithm is equal to the expected value of $\ln (x)$ :

$$
\ln (\bar{x})=\int \ln (x) \cdot f(x) d x=\int_{x_{0}}^{\infty} \ln (x) \cdot A \cdot x^{-\alpha} d x .
$$

This integral can be computed by integration by part; so, we get

$$
\begin{gathered}
\ln (\bar{x})=\left.\ln (x) \cdot \frac{A \cdot x^{1-\alpha}}{1-\alpha}\right|_{x_{0}} ^{\infty}-\int_{x_{0}}^{\infty} \frac{1}{x} \cdot \frac{A \cdot x^{1-\alpha}}{1-\alpha} d x= \\
\ln \left(x_{0}\right) \cdot \frac{A \cdot x_{0}^{-(\alpha-1)}}{\alpha-1}-\int_{x_{0}}^{\infty} \frac{A \cdot x^{-\alpha}}{1-\alpha} d x= \\
\ln \left(x_{0}\right) \cdot \frac{A \cdot x_{0}^{-(\alpha-1)}}{\alpha-1}-\left.\frac{A \cdot x^{-(\alpha-1)}}{(1-\alpha)^{2}}\right|_{x_{0}} ^{\infty}= \\
\ln \left(x_{0}\right) \cdot \frac{A \cdot x_{0}^{-(\alpha-1)}}{\alpha-1}+\frac{A \cdot x_{0}^{-(\alpha-1)}}{(1-\alpha)^{2}} .
\end{gathered}
$$

Substituting the expression $A=(\alpha-1) \cdot x_{0}^{\alpha-1}$ into this formula, we get

$$
\ln (\bar{x})=\ln \left(x_{0}\right)+\frac{1}{\alpha-1},
$$

hence

$$
\bar{x}=\exp (\ln (\bar{x}))=x_{0} \cdot \exp \left(\frac{1}{\alpha-1}\right)
$$

Comment. When $\alpha \rightarrow \infty$, the distribution tends to be concentrated on a single value $x_{0}-$ i.e., we have the case of absolute equality. In this case, as expected, all three characteristics - the mean, the median, and the new geometric mean - tends to the same value $x_{0}$. 


\section{Auxiliary Result: The New Measure of “Average” Income May Explain the Power-Law Character of Income Distribution}

In the previous section, we analyzed how the new measure of "average" income $\bar{x}=\exp \left(\int \ln (x) \cdot f(x) d x\right)$ behaves in situations when the income distribution follows a power law.

Interestingly, the power law itself can be derived based on this inequality measure. Indeed, suppose that all we know about the income distribution is the value $\bar{x}$, and the lower bound $\delta>0$ on possible incomes (this lower bound reflects the fact that a human being needs some minimal income to survive). There are many possible probability distributions $f(x)$ which are consistent with this information. In such situation, out of all such distributions, it is reasonable to select a one for which the entropy $S \stackrel{\text { def }}{=}-\int f(x) \cdot \ln (f(x)) d x$ is the largest; see, e.g., [10].

To find the distribution that maximizes the entropy $S$ under the constraints $\exp \left(\int \ln (x) \cdot f(x) d x\right)=\bar{x}$ and $\int f(x) d x=1$, we can use the Lagrange multiplier technique that reduces this constraint optimization problem to the unconstrained problem of optimizing a functional

$-\int f(x) \cdot \ln (f(x)) d x+\lambda_{1} \cdot\left(\exp \left(\int \ln (x) \cdot f(x) d x\right)-\bar{x}\right)+\lambda_{2} \cdot\left(\int f(x) d x-1\right)$,

for appropriate Lagrange multipliers $\lambda_{i}$. Differentiating this expression with respect to $f(x)$ and equating the derivative to 0 , we conclude that

$$
-\ln (f(x))-1+\lambda_{1} \cdot C \cdot \ln (x)+\lambda_{2}=0
$$

where $C \stackrel{\text { def }}{=} \exp \left(\int \ln (x) \cdot f(x) d x\right)$ and thus $C=\bar{x}$. Thus,

$$
\ln (f(x))=\left(\lambda_{2}-1\right)+\lambda_{1} \cdot \bar{x} \cdot \ln (x) .
$$

Applying the function $\exp (z)$ to both sides of this equality, we conclude that $f(x)=$ $A \cdot x^{-\alpha}$, where $A=\exp \left(\lambda_{2}-1\right)$ and $\alpha=-\lambda_{1} \cdot \bar{x}$. So, we indeed get the empirically observed power law for income distribution.

Acknowledgments. This work was supported in part by Chiang Main University, and also by the US National Science Foundation grants HRD-0734825 and HRD1242122 (Cyber-ShARE Center of Excellence) and DUE-0926721.

The authors are thankful to the anonymous referees for valuable suggestions.

\section{References}

1. E. F. Beckenbach and R. Bellman, Introduction to Inequalities, Mathematical Association of America, New York, 1975. 
2. J. Beirlant, Y. Goegevuer, J. Teugels, and J. Segers, Statistics of Extremes: Theory and Applications, Wiley, Chichester, 2004.

3. B. K. Chakrabarti, A. Chakraborti, and A. Chatterjee, Econophysics and Sociophysics: Trends and Perspectives, Wiley-VCH, Berlin, 2006.

4. A. Chatterjee, S. Yarlagadda, and B. K. Chakrabarti, Econophysics of Wealth Distributions, Springer-Verlag Italia, Milan, 2005.

5. J. D. Farmer and T. Lux (eds.). Applications of statistical physics in economics and finance, a special issue of the Journal of Economic Dynamics and Control, 2008, Vol. 32, No. 1, pp. 1320.

6. P. C. Fishburn, Nonlinear Preference and Utility Theory, John Hopkins Press, Baltimore, Maryland, 1988.

7. X. Gabaix, G. Parameswaran, P. Vasiliki, and H. E. Stanley, "Understanding the cubic and half-cubic laws of financial fluctuations", Physica A, 2003, Vol. 324, pp. 1-5.

8. C. P. Gomez and D. B. Shmoys, "Approximations and Randomization to Boost CSP Techniques", Annals of Operations Research, 2004, Vol. 130, pp. 117-141.

9. P. J. Huber, Robust Statistics, Wiley, Hoboken, New Jersey, 2004.

10. E. T. Jaynes, Probability Theory: The Logic of Science, Cambridge University Press, Cambridge, Massachisetts, 2003.

11. D. Kahneman, Thinking: Fast and Slow, Farrar, Straus, and Girroux, New York, 2011.

12. D. Kahneman and A. Tversky, "Advances in prospect theory: cumulative representation of uncertainty", Journal of Risk and Uncertainty, 1992, Vol. 5, pp. 297-324.

13. J. Kozielecki, Psychologicl decision theory, Kluwer, Dordrecht, Netherlands, 1981.

14. R. D. Luce and R. Raiffa, Games and Decisions: Introduction and Critical Survey, Dover, New York, 1989.

15. B. Mandelbrot, "The variation of certain speculative prices", J. Business, 1963, Vol. 36, pp. 394-419.

16. B. Mandelbrot, The Fractal Geometry of Nature, Freeman, San Francisco, California, 1983.

17. B. Mandelbrot and R. L. Hudson, The (Mis)behavior of Markets: A Fractal View of Financial Turbulence, Basic Books, 2006.

18. R. N. Mantegna and H. E. Stanley, An Introduction to Econophysics: Correlations and Complexity in Finance, Cambridge University Press, Cambridge, Massachusetts, 1999.

19. N. Markovich (ed.), Nonparametric Analysis of Univariate Heavy-Tailed Data: Research and Practice, Wiley, Chichester, 2007.

20. J. McCauley, Dynamics of Markets, Econophysics and Finance, Cambridge University Press, Cambridge, Massachusetts, 2004.

21. J. Nash, "The bargaining problem”, Econometrica, 1950, Vol. 18, No. 2, pp. 155-162.

22. H. T. Nguyen, V. Kreinovich, B. Wu, and G. Xiang, Computing Statistics under Interval and Fuzzy Uncertainty, Springer Verlag, Berlin, Heidelberg, 2012.

23. Organization for Economic Cooperation and Development (OECD), OECD Statistics, available at http://stats.oecd.org/

24. T. Piketty, Capital in the Twenty-First Century, Harvard University Press, Cambridge, Massachusetts, 2014.

25. S. T. Rachev and S. Mittnik, Stable Paretian Models in Finance, Wiley Publishers, New York, 2000.

26. H. Raiffa, Decision Analysis, McGraw-Hill, Columbus, Ohio, 1997.

27. S. I. Resnick, Heavy-Tail Phenomena: Probabilistic and Statistical Modeling, SpringerVarlag, New York, 2007.

28. B. Roehner, Patterns of Speculation - A Study in Observational Econophysics, Cambridge University Press, Cambridge, Massachusetts, 2002.

29. P. Rousseeuw and A. Leroy, Robust Regression and Outlier Detection, J. Wiley, New York, 1987.

30. D. J. Sheskin, Handbook of Parametric and Nonparametric Statistical Procedures, Chapman \& Hall/CRC, Boca Raton, Florida, 2011.

31. H. E. Stanley, L. A. N. Amaral, P. Gopikrishnan, and V. Plerou, "Scale invariance and universality of economic fluctuations", Physica A, 2000, Vol. 283, pp. 31-41. 
32. J. M. Steele, The Cauchy-Schwarz Master Class: An Introduction to the Art of Mathematical Inequalities, Cambridge University Press, New York, 2004.

33. S. V. Stoyanov, B. Racheva-Iotova, S. T. Rachev, and F. J. Fabozzi, "Stochastic models for risk estimation in volatile markets: a survey", Annals of Operations Research, 2010, Vol. 176, pp. 293-309.

34. P. Vasiliki and H. E. Stanley, "Stock return distributions: tests of scaling and universality from three distinct stock markets", Physical Review E: Statistical, Nonlinear, and Soft Matter Physics, 2008, Vol. 77, No. 3, Pt. 2, Publ. 037101. 